\title{
30.7 Tb/s (96x320 Gb/s) DP-32QAM transmission over 19-cell Photonic Band Gap Fiber
}

\author{
V.A.J.M. Sleiffer ${ }^{1}$, Y. Jung ${ }^{2}$, P. Leoni ${ }^{3}$, M. Kuschnerov ${ }^{4}$, N.V. Wheeler ${ }^{2}$, N. Baddela ${ }^{2}$, R.G.H. van Uden ${ }^{1}$, \\ C.M. Okonkwo', J.R. Hayes ${ }^{2}$, J. Wooler ${ }^{2}$, E. Numkam², R. Slavik' ${ }^{2}$, F. Poletti' ${ }^{2}$, M.N. Petrovich ${ }^{2}$, \\ V. Veljanovski ${ }^{4}$, S.U. Alam ${ }^{2}$, D.J. Richardson ${ }^{2}$, and H. de Waardt ${ }^{1}$ \\ ${ }^{1}$ COBRA institute, Eindhoven University of Technology, The Netherlands, e-mail: v.a.j.m.sleiffer@tue.nl \\ ${ }^{2}$ Optoelectronics Research Centre, University of Southampton, Southampton, SO17 1BJ, UK \\ ${ }^{3}$ Universität der Bundeswehr München, 85577 Neubiberg, Germany \\ ${ }^{4}$ Nokia Siemens Networks GmbH \& Co. KG, Munich, Germany
}

\begin{abstract}
We report for the first time coherently-detected, polarization-multiplexed transmission over a photonic band gap fiber. By transmitting 96 x 320-Gb/s DP-32QAM modulated channels, a net data rate of $24 \mathrm{~Tb} / \mathrm{s}$ was obtained.

OCIS codes: (060.1660) Coherent communications, (060.2330) Fiber optics communication, (060.4080) Modulation.
\end{abstract}

\section{Introduction}

Increasing the capacity per fiber to meet future bandwidth demands has recently increased research activity in one of the last dimensions left to exploit for multiplexing data, space. Space-division-multiplexing (SDM) is been exploited in two main research streams: multicore[1,2] and multimode transmission[3-5]. To date, the majority of work has focused on the use of solid core fibers and significant progress has been made. However, the fact remains that the nonlinear threshold, loss and bandwidth of these new fibers is approximately the same as for standard single-mode fiber (SSMF). Consequently, given the need for far more complex transmitters and receivers and the need for more complex fiber drawing technology, it is questionable as to whether the overall system costs will ever prove to be competitive to the deployment of N x SSMF systems. Significant benefits could be obtained from a fiber with lower loss and improved nonlinear tolerance relative to SSMF, allowing considerably increased fiber span lengths. Photonic band gap fiber (PBGF) has the potential to fulfill those requirements. Since most of the light is propagating in air, the nonlinear tolerance is greatly improved [6]. For the same reason, the ultimate minimum predicted losses are estimated to be significantly lower than those of SSMF [6]. Recently a record low-loss $(3.5 \mathrm{~dB} / \mathrm{km})$ 19-cell PBGF was presented [7]. Although this PBGF is heavily multimode, it was used by exciting only the fundamental mode to transmit a total capacity of $1.45 \mathrm{~Tb} / \mathrm{s}$ exploiting the full C-band using single-polarization OOK modulation [8]. Other research groups already demonstrated the possibility to use the PBGF for SDM using the same modulation type, although distances and data rates were very much lower $[9,10]$.

In this work we show for the first time coherently-detected, polarization-multiplexed transmission over a PBGF. For this experiment we used only the fundamental mode of the 19-cell PBGF reported in [7]. Using the extended C-band, we transmitted both 96x256-Gb/s dual-polarization (DP)-16QAM and 96x320-Gb/s DP-32QAM over the PBGF, obtaining, after accounting for 21\% of FEC overhead, net data rates of $19.2 \mathrm{~Tb} / \mathrm{s}$ and $24 \mathrm{~Tb} / \mathrm{s}$, respectively. This is by far the highest data-rate reported over PBGF to the best of our knowledge.

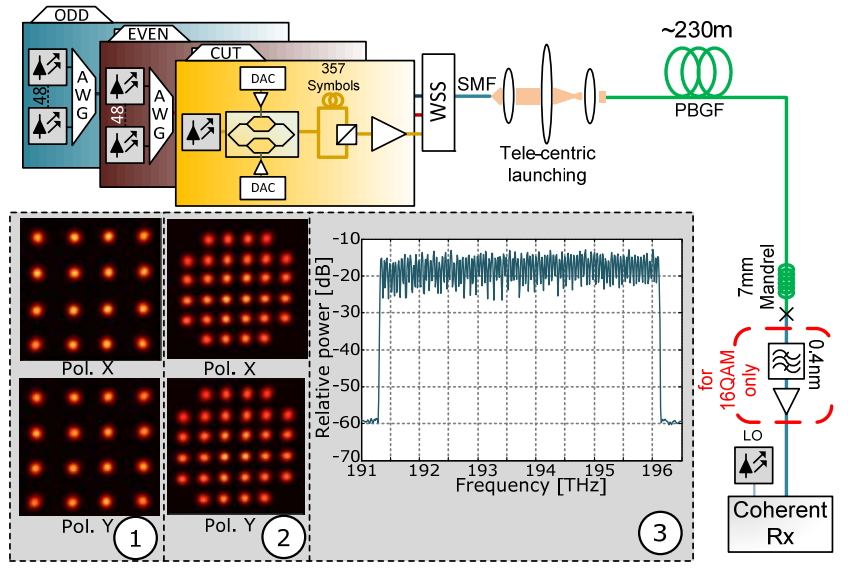

Fig. 1: Experimental Setup. (1) 16QAM constellation Back-toback, (2) 32QAM constellation Back-to-back. (3) Transmitted optical spectrum, 96 channels

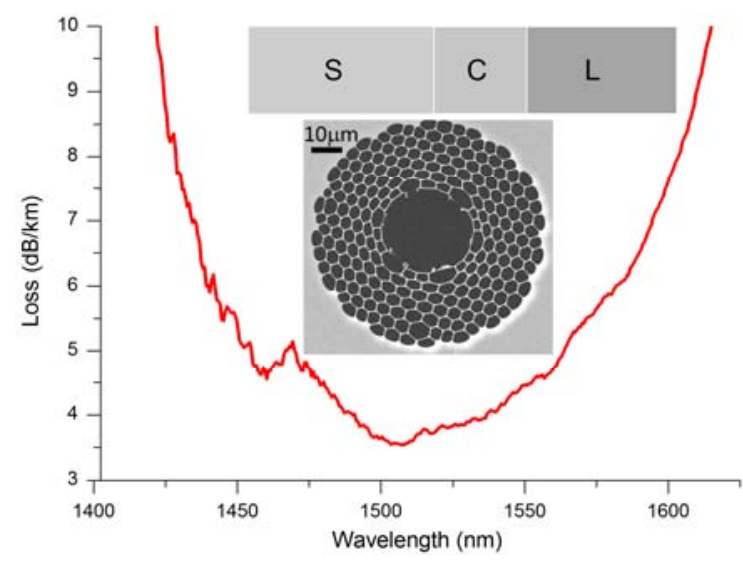

Fig. 2: Wavelength $[\mathrm{nm}]$ versus loss [dB/km]. Inset: 19-cell Photonic Band Gap fiber. 


\section{Experimental Setup}

Fig. 1 depicts the experimental setup. At the transmitter side, 96 channels carrying the 256-Gb/s DP-16QAM or 320-Gb/s DP-32QAM modulated signal were created. The 96 channels are divided in 48 even and 48 odd channels, of which one is replaced by the channel under test (CUT). The 48 even and odd channels were generated by multiplexing 48 ECL lasers running on the ITU 50-GHz grid ranging from $191.35 \mathrm{THz}$ to $196.1 \mathrm{THz}$, using AWGs. Subsequently the signals containing 48 wavelengths each are modulated using IQ-modulators. The modulators are driven by separate DACs for the in-phase and quadrature port, which were synchronized to generate the 16QAM or 32QAM symbols at 32GBaud. These symbols were created in the digital domain from regular PRBS15 sequences for 16QAM. Since 32QAM will have a higher error floor, 13.8\% soft-decision (SD)-FEC as described in [11, 12] was implemented as inner-code, yielding, assuming 7\% of hard-decision (HD)-FEC as outer-code, to a FEC-limit at a bit-error rate (BER) of $2 \cdot 10^{-2}$. The outputs of the DACs were electrically amplified before feeding them to the IQ-modulators. The output swing was set such that the IQ-modulators were operated in the linear regime, no pre-distortion was applied. After modulation the signals were polarization-multiplexed (POLMUX). This is achieved by splitting the signal into two equally powered tributaries, delaying one, and combining them again using a polarization-beam combiner. The CUT is effectively generated the same way, but only one laser was modulated. In the POLMUX stage of the CUT one of the polarization tributaries was delayed by 357 symbols with respect to the other. Finally, the three setups after de-correlation of several hundreds of symbols, are multiplexed using a wavelength-selective switch (WSS). One of the even or odd channels was dropped and the CUT is inserted at that wavelength.

The transmission link consists of 230m of the state-of-the-art 19-cell photonic band gap fiber (PBGF). The fiber and its wavelength dependent loss are shown in Fig. 2 [7]. Due to the fact that signals propagate in air rather than glass, this fiber offers a 30\% reduction in latency compared to SSMFs [8] and a considerable reduction of the nonlinear effects which ultimately limit the transmission distance of SSMFs. The 3 orders-of-magnitude lower nonlinearity of the fiber, combined with its large bandwidth $(160 \mathrm{~nm})$, are expected to allow in principle a higher transmission capacity than in SSMFs. Although the fiber used in this experiment supports multiple modes, for this proof of concept experiment data is only transmitted on the fundamental mode. To reduce crosstalk to higher-order modes, the launching into and detection from the PBGF must be very accurately controlled. In principle careful splicing of an SSMF at both the input and output of the PBGF can provide good crosstalk suppression. However, for additional control a free-space (tele-centric) launch (Fig. 1) is used. At the output of the PBGF an additional mode filter consisting of 60 turns around a $7 \mathrm{~mm}$ mandrel was applied to achieve a further improvement in high-order mode extinction. Although the fiber loss in the transmission band of interest was only $4.5 \mathrm{~dB} / \mathrm{km}$, the total span loss measured for this setup was $\sim 10 \mathrm{~dB}$, which we attribute to mode mismatch at both input and output end of the fiber and to the mode stripper section.

After transmission, the 256-Gb/s DP-16QAM the CUT was selected using a de-multiplexing filter. In case of 320-Gb/s DP-32QAM transmission, the CUT was selected using coherent channel selection in the coherent receiver. A single-ended commercial coherent receiver was used to receive the CUT which is connected to a $40 \mathrm{GSamples} / \mathrm{s}$ digital sampling scope. Afterwards the signal is demodulated using data-aided DSP. The results are obtained by averaging over at least two time-different shots, which contain 500,000 symbols for bit-error counting each.

\section{Results}

Fig. 3 shows the BER versus OSNR back-to-back curve and curve after transmission for single channel 256-Gb/s DP-16QAM at a 193.4-THz frequency. The implementation penalty of 256-Gb/s DP-16QAM at the FEC-limit is observed to be $2.2 \mathrm{~dB}$. For higher-order constellations with a high baudrate, like 16QAM and 32QAM at 32 GBaud, every kind of impairment (in this case crosstalk from higher-order modes) will have a lot of impact on the transmission performance [13]. Only a $0.5-\mathrm{dB}$ additional penalty at the FEC-limit is observed however for transmission of 256-Gb/s DP-16QAM over the 230m of PBGF, showing the successful suppression of crosstalk from higher-order modes.

In Fig. 4 the BER versus OSNR back-to-back and after transmission curve for single channel 320-Gb/s DP-32QAM are shown. A 4-dB implementation penalty is obtained for 320-Gb/s DP-32QAM. Fig. 4 additionally shows the curve after inner SD-FEC after transmission over the PBGF. Assuming an outer HD-FEC of

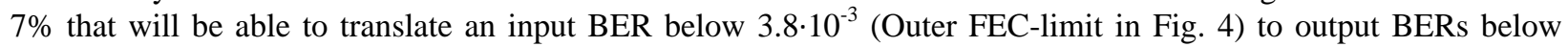
$1 \cdot 10^{-15}$ [14], the transmission is error free for an input BER of $2 \cdot 10^{-2}$. The transmission OSNR-penalty at the FEC-limit is only $0.4 \mathrm{~dB}$.

Fig. 5 shows the results obtained for all 96 channels after transmission over the PBGF for both 256-Gb/s DP-16QAM and 320-Gb/s DP-32QAM. The constellations for both modulation formats obtained after 


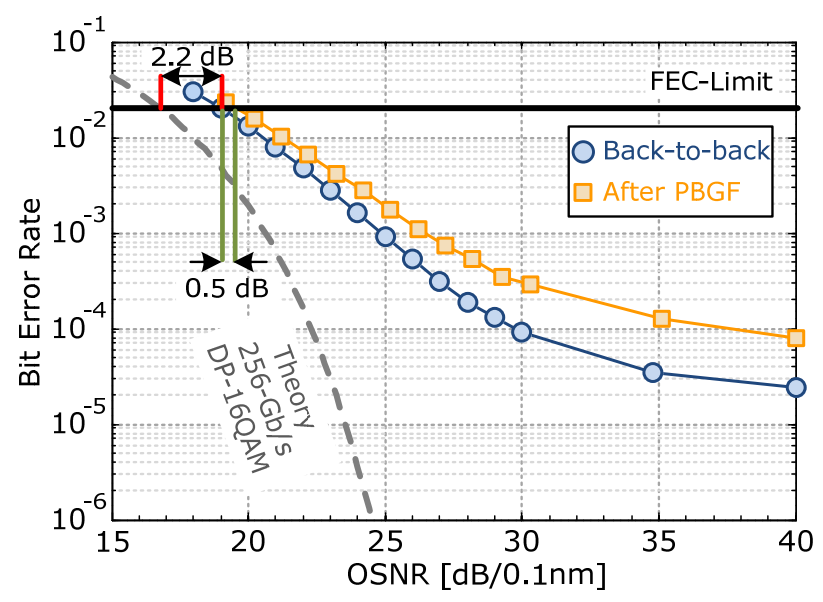

Fig. 3: Single channel (193.4 THz) 256-GB/s DP-16QAM BER vs. OSNR Back-to-back and after PBGF

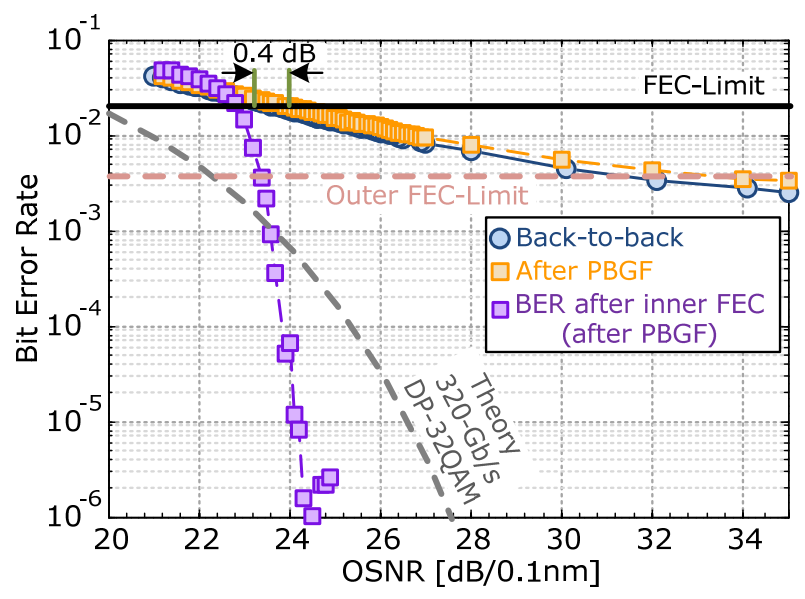

Fig. 4: Single channel (193.4 THz) 320-GB/s DP-32QAM BER vs. OSNR Back-to-back and after PBGF

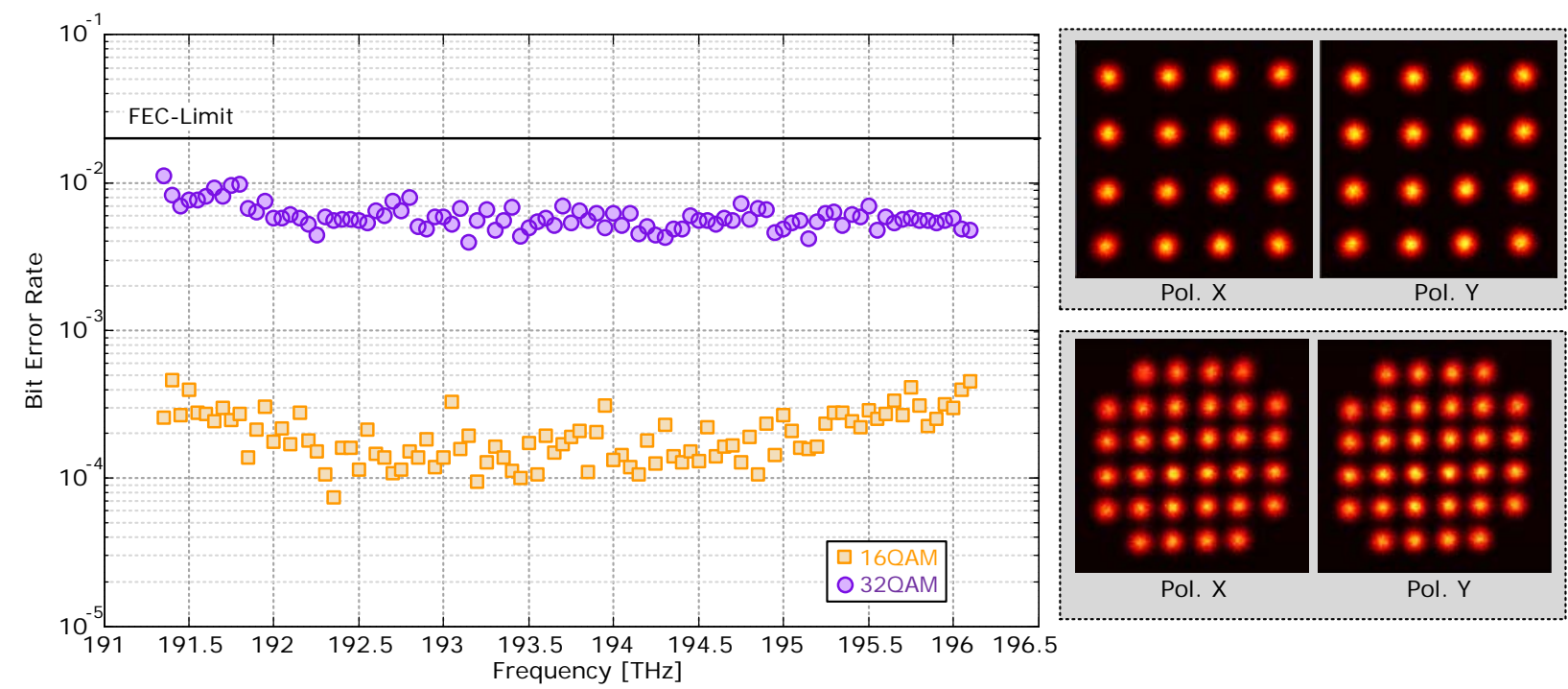

Fig. 5: BER vs. Frequency [THz]. Right: 16QAM and 32QAM constellations after transmission

transmission are shown on the right side of Fig. 5. In both cases the BERs are well below the FEC-limit. All 32QAM results showed zero post-inner-FEC errors.

\section{Conclusions}

We showed for the first time successful transmission of coherently-detected, polarization-multiplexed signals over photonic band gap fiber. We transmitted both 96x256-Gb/s DP-16QAM and 96x320-Gb/s DP-32QAM over 230m of 19-cell PBGF, obtaining, after accounting for 21\% of FEC overhead, net data rates of $19.2 \mathrm{~Tb} / \mathrm{s}$ and $24 \mathrm{~Tb} / \mathrm{s}$, respectively. By doing so, we showed, to the best of our knowledge, the highest data-rate transmission reported over PBGF. This shows the potential of PBGF being a broadband optical transmission fiber supporting high data-rates. Substantial higher transmission capacities should be possible by better exploiting the spectral bandwidth and the multimode property of the fiber.

This work was supported by the EU FP7-ICT MODE GAP project under grant agreement 258033.

\section{References}

[1] H. Takahara et al., Proc. ECOC'12, Th.3.C.1

[2] H. Takahashi et al., Proc. ECOC’12, Th.3.C.3

[3] S. Randel et al., Proc. OFC'12, PDP5C.5

[4] E. Ip et al., Proc. ECOC'11, Th.13.C.2

[5] V.A.J.M. Sleiffer et al., Proc. ECOC'12, Th.3.C.4

[6] M.N. Petrovich et al., Optics Express 16, 4337-4346 (2008)

[7] N. Wheeler et al., Proc. OFC’12, PDP5A.2
[8] R. Slavik et al., ECOC'12, Mo.2.F.2

[9] J. Carpenter et al., Proc. OFC'12, JW2A.41

[10] J. Xu et al., Proc. ECOC’11, We.10.P1.66

[11] R. M. Pyndiah, IEEE. Trans. on Comm. 46, 8 (1998)

[12] P. Leoni et al., Proc. SPPCom '12, SpTu3A.6

[13] P. Winzer et al., Proc. ECOC’11, Tu.5.B.7

[14] ITU-T Recommendation G.975.1, 2004, Appendix I.9. 